\begin{tabular}{|c|c|}
\hline Title & $\begin{array}{l}\text { Wounding stress induces phenylal anine ammonia ly ases, leading to the accumulation of pheny I propanoids in the model } \\
\text { liverwort Marchantia polymorpha }\end{array}$ \\
\hline Author(s) & Y oshikawa, Mayu; Luo, Weifeng; T anaka, Genta; Konishi, Y uka; Matsuura, Hideyuki; Takahashi, Kosaku \\
\hline Citation & $\begin{array}{l}\text { Phytochemistry, 155, 30-36 } \\
\text { https://doi.org/10.1016/.phytochem.2018.07.014 }\end{array}$ \\
\hline Issue Date & $2018-11$ \\
\hline Doc URL & http:/hdl. handle.net/2115/79700 \\
\hline Rights & $\begin{array}{l}\text { (9 2018. This manuscript version is made available under the CC-BY-NC-ND } 4.0 \text { license } \\
\text { http://reativecommons.org/icenses/by-nc-nd/4.0/ }\end{array}$ \\
\hline Rights(URL) & http://creativecommons.org/icenses/by-nc-nd/4.0/ \\
\hline Type & article (author version) \\
\hline File Information & MpPAL_20180719 Fig Supplement .pdf \\
\hline
\end{tabular}

Instructions for use 
Title

Wounding stress induces phenylalanine ammonia lyases, leading to the accumulation of phenylpropanoids in the model liverwort Marchantia polymorpha

\section{Author Names}

Mayu Yoshikawa, ${ }^{1}$ Weifeng Luo, ${ }^{1}$ Genta Tanaka, Yuka Konishi, Hideyuki Matsuura, Kosaku Takahashi*

Affiliations

Research Faculty of Agriculture, Hokkaido University, Sapporo, 060-8589, Japan

*To whom correspondence should be addressed. Tel.: +81-11-706-3349;

Fax: +81-11-706-2505; E-mail; kosaku@chem.agr.hokudai.ac.jp

${ }^{1}$ These authors contributed equally to this study. 


\section{Abstract}

Wounding stress induces the biosynthesis of various specialized metabolites in plants. In this study, wounding induced the biosynthesis of luteolin, apigenin, and isoriccardin C, which are biosynthesized through the phenylpropanoid pathway, in the model liverwort Marchantia polymorpha L (Marchantiaceae). Recombinant M. polymorpha phenylalanine ammonia lyases (MpPALs) exhibited PAL activity in vitro and converted phenylalanine into trans-cinnamic acid. Based on semi-quantitative RT-PCR analysis, the expression levels of the MpPAL genes were up-regulated after wounding. $\alpha$-Aminooxy- $\beta$-phenylpropionic acid, a PAL inhibitor, suppressed the production of wounding-induced phenolic compounds, luteolin, apigenin, and isoriccardin $\mathrm{C}$, in $M$. polymorpha. Thus, PAL is a committed step in the biosynthesis of phenylpropanoids in response to wounding in M. polymorpha. This study suggests that wound-induced specialized metabolites such as phenylpropanoids comprise a conserved defense system in land plants.

Keywords

Marchantia polymorpha; Marchantiaceae; Phenylalanine ammonia lyase; Phenylpropanoid; Wounding. 


\section{Introduction}

Plants are constantly subjected to environmental stresses; therefore, sophisticated mechanisms for adaptation to adverse environmental conditions have been developed. Because wounding by pathogens and insects can be fatal to plants, a system in plants spontaneously produces specialized metabolites to restrict pathogenic infection and insect attack in response to wounding, including antibacterial compounds and insect antifeedants (Ramakrishna and Ravishankar, 2011; War et al., 2012). Wounding stimulates the biosynthesis of various specialized metabolites in plants. Many of these bioactive plant specialized metabolites are also useful for humans as medicines and treatments for diseases. The activation of specialized metabolites following wounding is a practical and effective method to increase the concentrations of bioactive compounds.

Among the specialized metabolites produced by plants, phenolic compounds are widely distributed between algae and angiosperms, with important roles in plant physiology. Thus, plants produce a diverse array of phenolic compounds that are suitable for different stages of development and environments. These phenolic compounds are primarily classified into flavonoids, stilbenes, coumarins, monolignols, and lignans, among others, according to their chemical structures. Phenolic compounds have many biological functions and a great diversity of functions for plant defense, including UV absorbance, protection from pathogen and herbivore attack, allelopathic effects, cell wall reinforcement, and antioxidation (Kutchan et al., 2015).

Most phenolic compounds produced in plants are biosynthesized through the phenylpropanoid pathway, which begins with phenylalanine. Phenylalanine ammonia lyase (PAL) is the enzyme responsible for the conversion of phenylalanine into trans-cinnamic acid. PAL is the first and committed step in the phenylpropanoid pathway (Emiliani et al., 2009; Thomas, 2010). Plants respond to stress with alterations in PAL activity and phenylpropanoid accumulation (Dixon and Paiva, 1995; McConn et al., 1997). Wounding is a type of abiotic stress that induces PAL gene expression (Diallinas and Kanellis, 1994; Fukasawa-Akada et al., 1996) and elevates PAL activity 
(Kamo et al., 2000; Ke and Saltveit, 1989).

Bryophytes, such as liverworts, mosses and hornworts, are plants that are taxonomically intermediate between algae and vascular plants (Asakawa et al., 2013a; Asakawa et al., 2013b; Asakawa, 1982; Bowman et al., 2007; Qiu et al., 2006) and constitute an early, diverging lineage of land plants. Therefore, bryophytes are at a key position in plant evolution, and comprehension of the physiology of bryophytes should provide significant information on how plants colonized land (Bowman et al., 2007). Notably, the complete genome of the model liverwort Marchantia polymorpha has been sequenced and will provide insights into the evolutionary history of land plants (Bowman et al., 2017).

Bryophytes produce a wide variety of specialized metabolites; however, information regarding the mechanisms that regulate the induction of specialized metabolites in bryophytes is scarce. In the model moss Physcomitrella patens, UV-B irradiation triggers flavonoid biosynthesis and expression of the genes encoding chalcone synthases, important enzymes for flavonoid biosynthesis (Wolf et al., 2010). The moss Hypnum plumaeforme accumulates momilactones A and B, which were originally found as allelochemicals in rice in response to UV-C irradiation, elicitors, and $\mathrm{CuCl}_{2}$ (Kato-Noguchi, 2011; Okada et al., 2016). In the model liverwort Marchantia polymorpha L. (Marchantiaceae), UV-C irradiation induces bisbibenzyls, specialized metabolites typically produced in liverworts by the abscisic acid (ABA) signaling pathway (Kageyama et al., 2015). These studies suggest that activation of production of specialized metabolites in response to UV irradiation is a conserved defense mechanism against UV irradiation in land plants. Wounding, another serious environmental stress, induces volatile production in M. polymorpha (Kihara et al., 2015), suggesting that wounding may also act as a cue for specialized metabolite synthesis in M. polymorpha. Previous work suggests that specialized metabolites (volatiles) are defensive compounds in bryophytes and that wounding can stimulate specialized metabolite accumulation in M. polymorpha; thus, we might anticipate volatile production to be a defensive response to wounding. However, volatile production as a 
defensive response to wounding remains to be investigated in M. polymorpha.

In this study, wounding in M. polymorpha induced the expression of genes encoding PALs, which was accompanied by increases in phenylpropanoids such as a bisbibenzyl and flavonoids. Given that wounding stimulates the biosynthesis of specialized metabolites in flowering plants, wounding-induced specialized metabolite production is suggested to be a conserved physiological response in land plants.

\section{Results and Discussion}

\subsection{Identification of compounds induced by wounding}

The effects of wounding on the production of nonvolatile compounds were examined in $M$. polymorpha grown on 1/2 Gamborg's B5 agar medium. Plants were harvested at 1 hour after wounding, and metabolites were extracted with methanol. The resultant extract was analyzed by reversed-phase high-performance liquid chromatography (HPLC) (Figs. 1 and S1). Comparison of HPLC profiles between extracts of wounded and control plants revealed that wounding significantly increased the intensities of peaks $8,10,11,12$, and 13. Peaks 8 and 10 appeared only in the extract of the wounded plant. Thus, wounding induced the production of nonvolatile specialized metabolites in M. polymorpha. To identify the compounds induced by wounding in M. polymorpha, wounded plants $(510 \mathrm{~g})$ were soaked in methanol, and several steps of chromatography yielded compounds $\mathbf{1}$ (0.9 mg, peak 8), 2 (2.0 mg, peak 10), and 3 (33.3 mg, peak 11). All isolated compounds had potent UV absorbing capacity.

Based on high resolution field desorption mass spectrometry (HR-FDMS) analysis, the molecular formula of compound 1 was $\mathrm{C}_{15} \mathrm{H}_{10} \mathrm{O}_{6}$ (found at $\mathrm{m} / z$ 286.04823, calcd. 286.04774). For compound 1, the ${ }^{1} \mathrm{H}$ NMR spectrum revealed that all the proton signals were derived from $s p^{2}$ protons $\left(\delta_{\mathrm{H}} 6.5-7.5 \mathrm{ppm}\right)$. Analysis of the MS and NMR spectroscopic data (Supplemental data) determined that compound 1 was luteolin (Fig. 2) (Lee et al., 2013). 
From HR-FDMS spectral data, the molecular formula of compound $\mathbf{2}$ was determined to be $\mathrm{C}_{15} \mathrm{H}_{10} \mathrm{O}_{4}$ (found at $\mathrm{m} / \mathrm{z} 270.05392$, calcd. 270.05870). The ${ }^{1} \mathrm{H}$ NMR spectrum of compound 2 revealed that most proton signals were $s p^{2}$ proton signals $\left(\delta_{\mathrm{H}} 6.2-8.0 \mathrm{ppm}\right)$ and that the structure of compound $\mathbf{2}$ was similar to that of compound 1, which suggested that compound $\mathbf{2}$ was also a flavonoid. Analysis of the MS and NMR spectroscopic data (Supplemental data) revealed that compound 2 was apigenin (Fig. 2) (Lee et al., 2013). Production of luteolin (1) and apigenin (2) has been previously reported in M. polymorpha (Markham et al., 1998).

The molecular formula of compound 3 was $\mathrm{C}_{28} \mathrm{H}_{24} \mathrm{O}_{6}$ (found at $\mathrm{m} / z$ 424.16679, calculated 424.16746), based on HR-FDMS spectral data. The ${ }^{1} \mathrm{H}$ NMR spectrum of compound 3 revealed that most of its proton signals were derived from aromatic protons. Moreover, the characteristic olefin proton signal of bisbibenzyls, which was shifted at approximately $\delta_{\mathrm{H}} 5.5 \mathrm{ppm}$, was observed in the ${ }^{1} \mathrm{H}$ NMR spectrum of compound 3. Detailed investigations of the MS and NMR spectral data (Supplemental data) revealed that compound 3 was isoriccardin C (Fig. 2) (Asakawa et al., 1987). This compound is categorized as a bisbibenzyl, a characteristic secondary metabolite in liverworts.

These results showed that wounding induced the production of phenylpropanoids in $M$. polymorpha. Accordingly, a wounding-induced system for the biosynthesis of specialized metabolites is probably conserved in land plants. However, the structures of the compounds at peaks 12 and 13, which were not separated by any HPLC conditions in this study, could not be determined.

\subsection{Accumulation profiles of compounds $\mathbf{1}-\mathbf{3}$ after wounding}

Based on analysis of specialized metabolites in M. polymorpha, compounds 1-3 accumulated 1 hour after wounding. The time course of the accumulation of compounds 1-3 was examined by HPLC analysis. The concentrations of compounds 1-3 were transiently elevated at 1 and 2 hours after wounding and then decreased to 6 hours (Fig. 3). Because compounds $\mathbf{1}$ and $\mathbf{2}$ were not identified in non-wounded plants, the biosynthesis of compounds $\mathbf{1}$ and $\mathbf{2}$ was triggered after 
wounding. Therefore, when wounded, the concentrations of phenylpropanoids in M. polymorpha increased in a manner similar to that in flowering plants. Given that bisbibenzyls such as isoriccardin C (3) have antimicrobial activity (Asakawa et al., 1987), the transient increase of an antimicrobial compound is likely a rapid response to suppress pathogenic infection in M. polymorpha; moreover, the accumulation of an antimicrobial compound as a defense mechanism is probably conserved in land plants. However, the concentrations of compounds 1-3, which were at maximum levels at 1 and 2 hours after wounding, decreased 6 hours after wounding, suggesting that compounds 1-3 were further metabolized after reaching their maximum concentrations.

\subsection{Phenylalanine ammonia lyases in M. polymorpha}

Flavonoids and bisbibenzyls are categorized as phenylpropanoids, and the initial step in the biosynthesis of compounds $\mathbf{1}-\mathbf{3}$ is a PAL reaction, the non-oxidative deamination of phenylalanine to trans-cinnamic acid (Thomas, 2010). PALs have been extensively studied with respect to the production of natural products such as flavonoids, lignin, and a variety of other phenolic compounds in plants. However, no detailed studies of PAL activity in M. polymorpha have been conducted.

Genomic analysis predicted the presence of ten MpPAL genes in M. polymorpha (Bowman et al., 2017), which were designated MpPAL1 (Mapoly0014s0211.1), MpPAL2 (Mapoly0044s0114.1), MpPAL3 (Mapoly0009s0173.1), MpPAL4 (Mapoly0070s0061.1), MpPAL5 (Mapoly0070s0065.1), MpPAL6 (Mapoly0070s0068.1), MpPAL7 (Mapoly0070s0071.1), MpPAL8 (Mapoly0132s0049.1), MpPAL9 (Mapoly0005s0086.1), and MpPAL10 (Mapoly0005s0089.1). Similar to M. polymorpha, other land plants also have multiple $P A L$ genes (Bowman et al., 2017). The genes encoding cinnamate 4-hydroxylase $(\mathrm{C} 4 \mathrm{H})$ and 4-coumaroyl CoA ligase $(4 \mathrm{CL})$, which participate in phenylpropanoid biosynthesis, are also present in the genome of M. polymorpha. In contrast, no $P A L$, $\mathrm{C} 4 \mathrm{H}$, or $4 C L$ genes are present in algae such as Chlamydomonas, though they do have genes that encode enzymes in the shikimate pathway, which provides aromatic amino acids such as 
phenylalanine and tyrosine (Bowman et al., 2017). Based on genomic data from several plants, phenylpropanoids are considered to be characteristic secondary metabolites in land plants.

Phylogenetic analysis revealed that MpPAL2-10 formed a cluster that was separate from that of MpPAL1 and the other PALs of the model moss Physcomitrella patens and flowering plants (Fig. S2). These results suggested that MpPAL1 is an ancestral land plant PAL. Amino acid sequence alignment analysis of the MpPALs revealed that eight of them have the conserved active site amino acid sequence Ala-Ser-Gly (Fig. S3). Cyclization of Ala-Ser-Gly produces the cofactor of the PAL reaction, 3,5-dihydro-5-methylidene-4H-imidazole-4-one (MIO) (Schuster and J Rétey, 1995; Alunni et al., 2003; Calabrese et al., 2004). However, the predicted amino acid sequence of MpPAL10 is much shorter than that of the other predicted MpPALs and does not contain the conserved active site amino acid sequence Ala-Ser-Gly. Consequently, MpPAL10 likely does not have PAL activity. In the conserved amino acid sequence that forms MIO in MpPAL2, the Ser residue is substituted by Ala, a change that significantly reduced PAL activity in Petroselinum crispum L. (Schuster and Rétey, 1995); therefore, MpPAL2 likely has weak PAL activity.

\subsection{Enzymatic activity of the MpPALs}

To examine MpPAL activity, we attempted to clone nine of the candidate MpPAL genes; $\mathrm{MpPAL10}$ was excluded, as it lacks peptide sequence elements that are important for PAL activity. However, the cloning of two predicted genes, MpPAL5 and MpPAL9, failed due to low gene expression. The ORF of MpPAL6 is quite similar to that of MpPAL7; therefore, cloning MpPAL6 and MpPAL7 individually was difficult. The six other genes, MpPAL1, MpPAL2 MpPAL3, MpPAL4 $M p P A L 6$, and MpPAL8, were each inserted into a vector for recombinant protein synthesis, and the resultant vectors were transformed into E. coli. To evaluate the activity of each MpPAL, each E. coli strain overexpressing an $\mathrm{Mp} P A L$ was incubated with phenylalanine; subsequently, trans-cinnamic acid, the product of the PAL reaction, was analyzed using UPLC-MS/MS (Matsuura et al., 2009). 
The analytical data from the reaction mixtures containing the recombinant MpPALs showed clear peaks corresponding to trans-cinnamic acid (Fig. 4). In contrast, a trans-cinnamic acid peak was not found in a reaction mixture of the E. coli strain transformed with empty vector (Fig. 4). The peaks derived from trans-cinnamic acid in the reactions of MpPAL2 and MpPAL6 were smaller than those in the other MpPAL reactions. Due to the change in its functional amino acid sequence, MpPAL2 would reasonably show lower PAL activity. However, given that the peptide sequence of MpPAL6 is very similar to those of the other MpPALs, the expression of MpPAL6 may be lower than those of the other MpPALs in E. coli.

Therefore, the recombinant MpPALs tested in this study indeed exhibited PAL activity. As far as we know, this study is the first to demonstrate that a natural existing PAL in which the Ser residue in the conserved sequence that forms MIO is substituted by Ala (MpPAL2) has PAL activity.

\subsection{Wound-induced MpPAL expression}

To investigate the relationship between $\mathrm{Mp} P A L$ gene expression and wounding, semi-quantitative RT-PCR of the MpPALs was performed to analyze MpPAL expression. The results indicated that wounding transiently increased the expression of most $\mathrm{Mp} P A L \mathrm{~s}$, which were successively cloned in this study (Fig. 5). Accordingly, MpPAL gene expression was shown to correlate with the wounding-induced production of compounds 1-3. The data also suggest that flowering plants inherited the induction of $P A L$ gene expression in response to wounding from their common ancestor with bryophytes.

\subsection{Effect of a PAL inhibitor on the synthesis of compounds 1-3}

With the up-regulation of MpPALs after wounding, the concentrations of compounds 1-3 increased. To examine the relationship between PAL activity and the accumulation of compounds 13, the concentrations of compounds 1-3 were analyzed in $M$. polymorpha treated with a PAL 
inhibitor, $\alpha$-aminooxy- $\beta$-phenylpropionic acid (AOPP) (Amrhein and Gödeke, 1977), after wounding. Based on the analytical data, treatment with AOPP significantly suppressed the wounding-induced increase in the concentrations of compounds 1-3 (Fig. 6). Accordingly, the PAL reaction was demonstrated to be the first committed step in the biosynthesis of phenylpropanoids, i.e., compounds 1-3, in M. polymorpha. Considering that wounding induced $\mathrm{Mp} P A L$ gene expression, de novo synthesis of PAL proteins probably contributes to the accumulation of compounds $\mathbf{1 - 3}$ in $M$. polymorpha.

\subsection{ABA does not affect the accumulation of $\mathbf{1}-\mathbf{3}$}

In plants, wounding increases the concentrations of signaling compounds to trigger the biosynthesis of secondary metabolites. In a previous study, bisbibenzyls accumulated in $M$. polymorpha grown on agar supplemented with ABA (Kageyama et al., 2015). To examine whether ABA is involved in the rapid accumulation of compounds 1-3 after wounding, the ABA concentration in M. polymorpha after wounding was analyzed by UPLC-MS/MS (Kageyama et al., 2015). This analysis revealed that wounding did not increase the concentration of ABA within 6 hours in M. polymorpha (Fig. 7). Therefore, ABA is not involved in the wounding-induced rapid production of compounds 1-3 in M. polymorpha.

\subsection{2-oxo-phytodiemoic acid does not affect the accumulation of 1-3}

Wounding increases the concentration of 12-oxo-phytodiemoic acid (OPDA), an intermediate in the octadecanoid pathway, in M. polymorpha (Yamamoto et al., 2015). Therefore, the effect of OPDA on the biosynthesis of compounds 1-3 was investigated. HPLC analysis of M. polymorpha treated with $50 \mu \mathrm{M}$ for 1 hour revealed that OPDA did not significantly alter the concentration of compound 3 (Fig. 8); compounds 1 and $\mathbf{2}$ were not detected in OPDA-treated M. polymorpha (data not shown). These data suggested that OPDA did not play an important role in the 
wounding-induced biosynthesis of phenylpropanoids. Thus, it is highly likely that a signaling system activated by wounding that is not related to OPDA stimulates phenylpropanoid accumulation in $M$. polymorpha. In contrast to M. polymorpha, momilactones A and B biosynthesis is induced by OPDA in the moss $H$. plumaeforme (Okada et al., 2016), suggesting that mosses may have developed an OPDA signaling system to activate specialized metabolism in the process of plant evolution.

\section{Concluding Remarks}

In this study, wounding transiently induced the production of luteolin (1), apigenin (2) and isoriccardin C (3), which are biosynthesized through the phenylpropanoid pathway. Wounding also up-regulated the expression of MpPAL genes in M. polymorpha, and a PAL inhibitor suppressed the increase in compounds 1-3. The results of these different evaluations indicated that activation of the PAL reaction caused by wounding was important for the accumulation of phenylpropanoids in $M$. polymorpha. Because bisbibenzyls have antimicrobial activity, it is highly likely that the production of antimicrobial compounds induced by wounding is conserved as a defense system in land plants. As liverworts represent the most basal lineage of extant land plants, stress-induced specialized metabolite production may be a key mechanism to protect early land plants from environmental stresses.

\section{Experimental Methods}

\subsection{Plant materials}

Male Marchantia polymorpha L (Marchantiaceae), accession Takaragaike-1 (formally the 'male E line'), were asexually maintained and propagated through gemma growth, as described previously (Okada et al., 2000; Takenaka et al., 2000). Takaragaike accessions were selected for sequencing the genome of M. polymorpha (Bowman et al., 2017). Plants were propagated on 1/2 Gamborg's B5 medium containing $1.4 \%$ agar medium under $50-60 \mu \mathrm{mol}$ photons $\mathrm{m}^{-2} \mathrm{~s}^{-1}$ of continuous fluorescent 
light at $22^{\circ} \mathrm{C}$ unless otherwise stated.

\subsection{Wounding and chemical treatments}

Marchantia polymorpha plants, which were grown on 1/2 Gamborg's B5 medium containing $1.4 \%$ agar medium for 30 days, were wounded by pressing with tweezers and were then harvested at the indicated time after wounding. For 12-oxo-phytodienoic acid (OPDA) treatment, approximately $0.3 \mathrm{ml}$ of $50 \mu \mathrm{M}$ OPDA was sprayed onto plants grown in the conditions described above, and the plants were then harvested after 1 hour. OPDA was synthesized according to the method of Kajiwara et al. (2012). For treatment with $\alpha$-aminooxy- $\beta$-phenylpropionic acid (AOPP, Wako pure chemicals, Osaka, Japan), approximately $0.3 \mathrm{ml}$ of $1 \mathrm{mM}$ AOPP was sprayed onto plants grown in the conditions described above, and then the plants were harvested at the indicated time after treatment.

\subsection{HPLC analysis of constituents in M. polymorpha after wounding}

Marchantia polymorpha was grown on 1/2 Gamborg's B5 medium containing $1.4 \%$ agar for 30 days. After wounding, plants were harvested at the indicated time and were then frozen in liquid nitrogen. Samples (approximately $200 \mathrm{mg}$ ) of frozen plants were crushed and extracted with $\mathrm{MeOH}$ (1 ml) with 2,4,6-trihydroxyacetophenone $(0.2 \mu \mathrm{g})$ as the internal standard. After filtration and evaporation, the MeOH extract was analyzed by reversed-phase HPLC (column; InertSustain C18, $10 \mathrm{~mm}$ i.d. $\times 250 \mathrm{~mm}$, GL Sciences, Tokyo, Japan; UV, $220 \mathrm{~nm}$; flow, $3.0 \mathrm{ml} / \mathrm{min}$ ) with mixed solvents of $\mathrm{MeOH}$ and $\mathrm{H}_{2} \mathrm{O}$ using a linear gradient mode in which the gradient of $\mathrm{MeOH}$ with $0.05 \% \mathrm{AcOH}$ to $\mathrm{H}_{2} \mathrm{O}$ with $0.05 \% \mathrm{AcOH}$ was held at $20: 80$ from 0 min to $5 \mathrm{~min}$ and then increased linearly from $5 \mathrm{~min}$ to $25 \mathrm{~min}$ to 100:0. The gradient of 100:0 was maintained from $25 \mathrm{~min}$ to $30 \mathrm{~min}$. Concentrations of compounds 1-3 were calculated based on the peak area of standard compounds.

\subsection{Isolation of compounds $\mathbf{1}-\mathbf{3}$}


Marchantia polymorpha plants were grown on 1/2 Gamborg's B5 medium containing $1.4 \%$ agar. Plants $(510 \mathrm{~g})$ were harvested at 1 hour after wounding, frozen in liquid nitrogen, crushed, and soaked in $\mathrm{MeOH}$ (3 1). After filtration and evaporation of the $\mathrm{MeOH}$ extract, the obtained residue (43 g) was dissolved in $\mathrm{MeOH}^{-} \mathrm{H}_{2} \mathrm{O}(9: 1, \mathrm{v} / \mathrm{v})$ and then partitioned with $n$-hexane. The resultant $\mathrm{MeOH}^{-}$ $\mathrm{H}_{2} \mathrm{O}$ layer $(9: 1, \mathrm{v} / \mathrm{v})$ was concentrated in vacuo, and the obtained residue was partitioned with EtOAc and $\mathrm{H}_{2} \mathrm{O}$. After evaporation of the EtOAc extract, the residual extract ( $1.0 \mathrm{~g}$ ) was loaded onto a silica gel column chromatography and separated with a mixed solvent of $\mathrm{MeOH}$ and $\mathrm{CHCl}_{3}$ in which the $\mathrm{MeOH}$ ratio was increased gradually to obtain fractions 1 to 6 . Fraction 3 (168 mg), which was eluted with a mixed solvent of $\mathrm{MeOH}-\mathrm{CHCl}_{3}(1: 9)$, was separated by reversed-phase HPLC (column; InertSustain C18, 10 mm i.d. $\times 250$ mm, GL Sciences, Tokyo, Japan; UV, $220 \mathrm{~nm}$; solvent, $60 \% \mathrm{MeOH}$ aqueous solution; flow, $3.0 \mathrm{ml} / \mathrm{min})$ to yield compounds $1(0.9 \mathrm{mg})$ and $2(2.0 \mathrm{mg})$. Fraction $2(227 \mathrm{mg})$, which was eluted with a mixed solvent of $\mathrm{MeOH}-\mathrm{CHCl}_{3}(5: 95)$, was separated by reversed-phase HPLC (column; InertSustain C18, $10 \mathrm{~mm}$ i.d. $\times 250 \mathrm{~mm}$, GL Sciences, Tokyo, Japan; UV, $220 \mathrm{~nm}$; solvent, $85 \% \mathrm{MeOH}$ aqueous solution; flow, $3.0 \mathrm{ml} / \mathrm{min}$ ) to yield compound 3 (33.3 mg).

\subsection{Identification of structures $\mathbf{1}-\mathbf{3}$}

The ${ }^{1} \mathrm{H}$ NMR and ${ }^{13} \mathrm{C}$ NMR spectra were recorded on a Bruker AMX-500 NMR spectrometer (Bruker, Karlsruhe, Germany). ${ }^{1} \mathrm{H}$ NMR chemical shifts were referenced to the residual $\mathrm{CDCl}_{3}$ solvent peak at $\delta 7.24 \mathrm{ppm}$, and ${ }^{13} \mathrm{C}$ NMR chemical shifts were referenced to the $\mathrm{CDCl}_{3}$ solvent peak at $\delta 77.0 \mathrm{ppm}$. HR-FDMS results were recorded on a JEOL JMS T100GCV mass spectrometer (Jeol, Tokyo, Japan). The NMR spectroscopic data of compounds 1-3 are shown in the Supplemental data.

\section{6. Cloning of the MpPALs}

Total RNA of $M$. polymorpha (ca. $100 \mathrm{mg}$ ) was extracted using a conventional 
phenol-chloroform method. Reverse transcription (M-MLV Reverse Transcriptase, Invitrogen, Carlsbad, CA, USA) was performed according to the manufacturer's instructions to generate cDNA. The primer set for each MpPAL gene was used to amplify its ORF. Each PCR was performed in a 40- $\mu 1$ volume per tube, containing $3 \mu \mathrm{l}$ of dNTP mixture (2.0 mM each), $3 \mu$ of each primer $(5 \mu \mathrm{M})$, $1 \mu \mathrm{l}$ of cDNA, $20 \mu \mathrm{l}$ of $2 \times$ PCR buffer, $0.5 \mu \mathrm{l}$ of KOD FX DNA polymerase (Toyobo, Japan) and 9.5 $\mu \mathrm{l}$ of Milli-Q water according to the manufacturer's instructions. The obtained PCR products were purified and inserted individually into the pBlueScript SK II (+) vector (Stratagene, USA), which was digested with $E c o R \mathrm{~V}$ (Takara, Japan). DNA sequencing was performed to confirm that the target gene was successfully inserted into the plasmid. The primers used in this study are listed in Table S1.

4.7. Construction of $E$. coli strains with the $M p P A L$ overexpression vectors

PCR was performed in $20-\mu 1$ reaction mixtures containing $2 \mu 1$ of a dNTP mixture $(2.0 \mathrm{mM}$ each dNTP), $10 \mu \mathrm{l}$ of $2 \times$ PCR buffer, $1 \mu 1$ of each primer $(5 \mu \mathrm{M}), 1 \mu 1$ of pBluescript SK $(+)$ containing an $\operatorname{MpPAL}(200 \mathrm{ng} / \mu \mathrm{l}), 0.5 \mu \mathrm{l}$ of KOD FX DNA polymerase (Toyobo, Japan), and $4.5 \mu \mathrm{l}$ of Milli-Q water. Each PCR was performed according to the manufacturer's instructions. The PCR product then was purified and ligated into the pET23a vector (Merck, USA) using Ligation Mix (Takara, Japan) according to the standard procedure. Each constructed pET23a plasmid carrying an MpPAL gene was transformed in E. coli BL21 using a standard procedure. The primers used in this study are listed in Table S2.

\subsection{PAL activity of the recombinant MpPALs}

E. coli cells carrying the overexpression vector pET23a containing each of the MpPALs were respectively cultured overnight in $5 \mathrm{ml}$ of LB medium containing $50 \mu \mathrm{g} / \mathrm{ml}$ of ampicillin at $37^{\circ} \mathrm{C}$. The culture was transferred into fresh LB medium $(2 \mathrm{ml})$ and incubated until the absorbance at 600 $\mathrm{nm}$ reached 0.2 . Then, isopropyl thio- $\beta$-D-galactoside (IPTG) was added to a final concentration of 1 
$\mathrm{mM}$, and the culture was incubated at $37^{\circ} \mathrm{C}$ for 5 hours to induce protein production. The cells were collected by centrifugation $\left(7000 \times \mathrm{g}, 5 \mathrm{~min}, 4^{\circ} \mathrm{C}\right)$, suspended in $2 \mathrm{ml}$ of $0.1 \mathrm{M}$ sodium borate buffer $(\mathrm{pH} 8.8)$, and disrupted by ultra-sonication. After centrifugation $\left(12000 \times g, 10 \mathrm{~min}, 4^{\circ} \mathrm{C}\right)$, the supernatant was used as an enzyme extract. One milliliter of each enzyme extract was pre-incubated at $40^{\circ} \mathrm{C}(5 \mathrm{~min})$ and then the reaction was started by adding $200 \mu \mathrm{l}$ of $0.1 \mathrm{M}$ sodium borate buffer (pH 8.8) containing phenylalanine $(10 \mathrm{mg} / \mathrm{ml})$. After 5 hours of incubation at $40^{\circ} \mathrm{C}$, the reaction was stopped by adding $100 \mu \mathrm{L}$ of $5 \mathrm{M} \mathrm{HCl}$. Trans-cinnamic acid in the reaction mixtures was analyzed according to the method of Matsuura et al. (2009).

\subsection{Semi-quantitative RT-PCR of the MpPALs}

M. polymorpha plants were grown on 1/2 Gamborg's B5 medium containing $1.4 \%$ agar for 30 days and then wounded. After 0, 1, 2 and 6 hours, total RNA was isolated using an innuPREP Plant RNA kit (Analytik Jena AG, Germany). Total RNA $(2 \mu \mathrm{g})$ was used as the template for cDNA synthesis. First strand cDNA was synthesized with M-MLV Reverse Transcriptase (Invitrogen) and oligo (dT) primers according to the manufacturer's instructions. To estimate the expression levels of the MpPALs, the PCR was optimized for a $12.5-\mu 1$ reaction mixture containing $1.25 \mu \mathrm{l}$ of dNTP mixture ( $2.0 \mathrm{mM}$ each), $0.75 \mu \mathrm{l}$ of each primer $(10 \mu \mathrm{M}), 0.25 \mu \mathrm{l}$ of cDNA, $6.25 \mu \mathrm{l}$ of $2 \times$ PCR buffer, $0.25 \mu \mathrm{l}$ of KOD FX DNA polymerase (Toyobo, Japan) and $3 \mu \mathrm{l}$ of Milli-Q water according to the manufacturer's instructions. The PCR was carried out according to the manufacturer's instructions. The primers are listed in Table S1. The annealing temperatures and the number of PCR cycles are shown in Table S3. The PCR products were analyzed by gel electrophoresis and visualized after ethidium bromide staining.

\subsection{UPLC-MS/MS analysis of ABA in M. polymorpha}

Plants with or without wounding treatment (approximately $1.0 \mathrm{~g}$ ) were frozen with liquid 
nitrogen, crushed, and soaked overnight in EtOH $(15 \mathrm{ml})$. UPLC analysis of ABA was conducted according to the method of Kageyama et al. (2015).

\section{Acknowledgments}

We are grateful to Dr. E. Fukushi and Mr. Takata of Hokkaido University for measurement of the mass spectral data. We appreciate Dr. Kohchi of Kyoto University and Dr. Ishizaki of Kobe University for giving valuable advice during our study. This study was financially supported by Hokkaido University. We used the UPLC-MS/MS system of the Research Faculty of Agriculture, Hokkaido University.

\section{References}

Alunni, S., Cipiciani, A., Fioroni, G., Ottavi, L., 2003. Mechanisms of inhibition of phenylalanine ammonia-lyase by phenol inhibitors and phenol/glycine synergistic inhibitors. Arch. Biochem. Biophys. 412, 170-175.

Amrhein, N., Gödeke, K.H., 1977. $\alpha$-aminooxy- $\beta$-phenylpropionic acid — a potent inhibitor of L-phenylalanine ammonia-lyase in vitro and in vivo. Plant Sci. Lett. 8, 313-317.

Asakawa, Y., 1982. Chemical constituents of the Hepaticae, in: Herz, W., Grisebach, H., Kirbt, G.W. (Eds.), Progress in the Chemistry of Organic Natural Products, vol. 42. Springer-Verlag, Vienna, Austria, pp. 1-286.

Asakawa, Y., Ludwiczuk, A., Nagashima, F., 2013a. Phytochemical and biological studies of bryophytes. Phytochemistry $91,52-80$.

Asakawa, Y., Ludwiczuk, A., Nagashima, F., 2013b. Chemical constituents of the bryophytes, in: Kinghorn, A.D., Falk, H., Kobayashi, J. (Eds.), Progress in the Chemistry of Organic Natural Products, vol. 95. Springer-Verlag, Vienna, Austria, pp. 1-796. 
Asakawa, Y., Tori, M., Takikawa, K., Krishnamurty, H.G., Kar, S.K., 1987. Cyclic bis(bibenzyls) and related compounds from the liverworts marchantia polymorpha and marchantia palmata. Phytochemistry 26, 1811-1816.

Bowman, J.L., Floyd, S.K., Sakakibara, K., 2007. Green genes-comparative genomics of the green branch of life. Cell 129, 229-234.

Bowman, J.L., Kohchi, T., Yamato, K.T., Jenkins, J., Shu, S., Ishizaki, K., Yamaoka, S., Nishihama, R., Nakamura, Y., Berger, F., Adam, C., Aki, S.S., Althoff, Fl, Araki, T., Arteaga-Vazquez, M.A., Balasubrmanian, S., Barry, K., Bauer, D., Boehm, C.R., Briginshaw, L., Caballero-Perez, J., Catarino, B., Chen, F., Chiyoda, S., Chovatia, M., Davies, K.M., Delmans, M., Demura, T., Dierschke, T., Dolan, L., Dorantes-Acosta, A.E., Eklund, D.M., Florent, S.N., Flores-Sandoval, E., Fujiyama, A., Fukuzawa, H., Galik, B., Grimanelli, D., Grimwood, J., Grossniklaus, U., Hamada, T., Haseloff, J., Hetherington, A.J., Higo, A., Hirakawa, Y., Hundley, H.N., Ikeda, Y., Inoue, K., Inoue, S.I., Ishida, S., Jia, Q., Kakita, M., Kanazawa, T., Kawai, Y., Kawashima, T., Kennedy, M., Kinose, K., Kinoshita, T., Kohara, Y., Koide, E., Komatsu, K., Kopischke, S., Kubo, M., Kyozuka, J., Lagercrantz, U., Lin, S.S., Lindquist, E., Lipzen, A.M., Lu, C.W., De Luna, E., Martienssen, R.A., Minamino, N., Mizutani, M., Mizutani, M., Mochizuki, N., Monte, I., Mosher, R., Nagasaki, H., Nakagami, H., Naramoto, S., Nishitani, K., Ohtani, M., Okamoto, T., Okumura, M., Phillips, J., Pollak, B., Reinders, A., Rövekamp, M., Sano, R., Sawa, S., Schmid, M.W., Shirakawa, M., Solano, R., Spunde, A., Suetsugu, N., Sugano, S., Sugiyama, A., Sun, R., Suzuki, Y., Takenaka, M., Takezawa, D., Tomogane, H., Tsuzuki, M., Ueda, T., Umeda, M., Ward, J.M., Watanabe, Y., Yazaki, K., Yokoyama, R., Yoshitake, Y., Yotsui, I., Zachgo, S., Schmutz, J., 2017. Insights into land plant evolution garnered from the marchantia polymorpha genome. Cell 171, 287-304. 
Calabrese, J.C., Jordan, D.B., Boodhoo, A., Sariaslani, S., Vannelli, T., 2004. Crystal structure of phenylalanine ammonia lyase: multiple helix dipoles implicated in catalysis. Biochemistry 43, 11403-11416.

Diallinas, G., Kanellis, A.K., 1994. A phenylalanine ammonia-lyase genefrom melon fruit: cDNA cloning, sequence and expression inresponse to development and wounding. Plant Mol. Biol. $26,473-479$.

Dixon, R.A., Paiva, N.L., 1995. Stress-induced phenylpropanoid metabolism. Plant Cell 7, 10851097.

Emiliani, G., Fondi, M., Fani, R., Gribaldo, S., 2009. A horizontal gene transfer at the origin of phenylpropanoid metabolism: a key adaptation of plants to land. Biol. Direct 4. doi:10.1186/1745-6150-4-7.

Fukasawa-Akada, T., Kung, S.D., Watson, J.C., 1996. Phenylalanine ammonia-lyase gene structure, expression, and evolution in nicotiana. Plant Mol. Biol. 30, 711-722.

Kageyama, A., Ishizaki, K., Kohchi, T., Matsuura, H., Takahashi, K., 2015. Abscisic acid induces biosynthesis of bisbibenzyls and tolerance to UV-C in the liverwort marchantia polymorpha. Phytochemistry 117, 547-553.

Kamo, T., Hirai, N., Tsuda, M., Fujioka, D., Ohigashi, H., 2000. Changes in the content and biosynthesis of phytoalexins in banana fruit. Biosci. Biotechnol. Biochem. 64, 2089-2098.

Kato-Noguchi, H., 2011. Convergent or parallel molecular evolution of momilactone A and B: potent allelochemicals, momilactones have been found only in rice and the moss hypnum plumaeforme. J. Plant Physiol. 168, 1511-1516.

Ke, D., Saltveit, M.E., 1989. Wound-induced ethylene production, phenolic metabolism and susceptibility to russet spotting in iceberg lettuce. Physiol. Plant. 76, 412-418.

Kihara, H., Tanaka, M., Yamato, K.T., Horibata, A., Yamada, A., Kita, S., Ishizaki, K., Kajikawa, M., Fukuzawa, H., Kohchi, T., Akakabe, Y., Matsui, K., 2014. Arachidonic acid-dependent 
carbon-eight volatile synthesis from wounded liverwort (marchantia polymorpha). Phytochemistry 107, 42-49.

Kutchan, T.M., Gershenzon, J., Møller, B.L., Gang, D.R., 2015. Natural products, in: Buchanan, B., Gruissem, W., Jones, R.L. (Eds.), Biochemistry and Molecular Biology of Plants, Second Edition. John Wiley and Sons, Ltd., New Jersey, pp. 1131-1206.

Lee, J.H., Park, K.H., Lee, M.H., Kim, H.T., Seo, W.D., Kim, J.Y., Baek, I.Y., Jang, D.S., Ha, T.J., 2013. Identification, characterisation, and quantification of phenolic compounds in the antioxidant activity-containing fraction from the seeds of Korean perilla (perilla frutescens) cultivars. Food Chem. 136, 843-852.

Markham, K.R., Ryan, K.G., Bloor, S.J., Mitchell, K.A., 1998. An increase in the luteolin: apigenin ratio in marchantia polymorpha on UV-B enhancement. Phytochemistry 48, 791-794.

Matsuura, H., Aoi, A., Satou, C., Nakaya, M., Masuta, C., Nabeta, K., 2009. Simultaneous UPLC MS/MS analysis of endogenous jasmonic acid, salicylic acid, and their related compounds. Plant Growth Regul. 57, 293-301.

McConn, M., Creelman, R.A., Bell, E., Mullet, J.E., Browse, J., 1997. Jasmonate is essential for insect defense in Arabidopsis. Proc. Natl Acad. Sci. U.S.A. 94, 5473-5477.

Okada, K., Kawaide, H., Miyamoto, K., Miyazaki, S., Kainuma, R., Kimura, H., Fujiwara, K., Natsume, M., Nojiri, H., Nakajima, M., Yamane, H., Hatano, Y., Nozaki, H., Hayashi, K., 2016. HpDTC1, a stress-inducible bifunctional diterpene cyclase involved in Momilactone biosynthesis, functions in chemical defence in the Moss hypnum plumaeforme. Sci. Rep. 6 , Article number: 25316, 25316. doi:10.1038/srep25316.

Okada, S., Fujisawa, M., Sone, T., Nakayama, S., Nishiyama, R., Takenaka, M., Yamaoka, S., Sakaida, M., Kono, K., Takahama, M., Yamato, K.T., Fukuzawa, H., Brennicke, A., Ohyama, K., 2000. Construction of male and female PAC genomic libraries suitable for identification 
of Y-chromosome-specific clones from the liverwort, Marchantia polymorpha. Plant J., 24, $421-428$

Qiu, Y.L., Li, L., Wang, B., Chen, Z., Knoop, V., Groth-Malonek, M., Dombrovska, O., Lee, J., Kent, L., Rest, J., Estabrook, G.F., Hendry, T.A., Taylor, D.W., Testa, C.M., Ambros, M., Crandall-Stotler, B., Duff, R.J., Stech, M., Frey, W., Quandt, D., Davis, C.C., 2006. The deepest divergences in land plants inferred from phylogenomic evidence. Proc. Natl. Acad. Sci. U.S.A. 103, 15511-15516.

Ramakrishna A, Ravishankar G.A., 2011. Influence of abiotic stress signals on secondary metabolites in plants. Plant Signal Behav. 6, 1720-1731.

Schuster, B., Rétey, J., 1995. The mechanism of action of phenylalanine ammonia-lyase: the role of prosthetic dehydroalanine. Proc. Natl. Acad. Sci. U. S. A. 92, 8433-8437.

Takenaka, M., Yamaoka, S., Hanajiri, T., Shimizu-Ueda, Y., Yamato, K.T., Fukuzawa, H., Ohyama, K., 2000. Direct transformation and plant regeneration of the haploid liverwort Marchantia polymorpha L. Transgenic Res. 9, 179-185.

War, A.R., Paulraj, M.G., Ahmad, T., Buhroo, A.A., Hussain, B., Ignacimuthu, S., Sharma, H.C., 2012. Mechanisms of plant defense against insect herbivores. Plant Signal Behav. 7, 1306-1320.

Thomas, V., 2010. Phenylpropanoid biosynthesis. Mol. Plant 3, 2-20.

Yamamoto, Y., Ohshika, J., Takahashi, T., Ishizaki, K., Kohchi, T., Matusuura, H., Takahashi, K., 2015. Functional analysis of allene oxide cyclase, MpAOC, in the liverwort marchantia polymorpha. Phytochemistry 116, 48-56. 
Figure legends

Fig. 1. HPLC chromatograms of the M. polymorpha extract with or without wounding treatment.

Upper chromatogram, control plant; lower chromatogram, wounded plant. Plants were grown on $1 / 2$ Gamborg's B5 medium containing $1.4 \%$ agar at $22^{\circ} \mathrm{C}$ under continuous white fluorescent light for 30 days. Plants were harvested at 1 hour after wounding. HPLC conditions are described in the Experimental Methods.

Fig. 2. The structures of luteolin (1), apigenin (2), and isoriccardin C (3).

Fig. 3. Accumulation of luteolin (1), apigenin (2), and isoriccardin C (3) induced by wounding in $M$. polymorpha.

M. polymorpha plants were grown on 1/2 Gamborg's B5 medium containing $1.4 \%$ agar for 30 days and then subjected to wounding treatment. Plants were harvested at the indicated time. Data represent means \pm S.D. of three separate experiments. The asterisks represent significant differences between wounded and control plants (Student's $t$-test, ${ }^{*} p<0.05,{ }^{* *} p<0.01$ ).

Fig. 4. In vitro PAL activity of the recombinant MpPALs.

A product ion peak at $m / z 108.00$ that was derived from the precursor ion peak of trans-cinnamic acid- $d_{6}$ at $\mathrm{m} / z 152.97[\mathrm{M}-\mathrm{H}]^{-}$and a product ion peak at $\mathrm{m} / \mathrm{z} 102.40$ that was derived from the precursor ion peak of trans-cinnamic acid at $m / z 146.71[\mathrm{M}-\mathrm{H}]^{-}$were analyzed in the MRM mode of UPLC-MS/MS. Standard: chromatogram of internal standard trans-cinnamic acid- $d_{6}$; vector control: 
chromatogram of the reaction mixture with $E$. coli containing empty pET23a.

Fig. 5. Effects of wounding on $M p P A L$ gene expression.

M. polymorpha plants were grown on 1/2 Gamborg's B5 medium containing $1.4 \%$ agar for 30 days and then subjected to wounding treatment. Plants were harvested at the indicated times. The expression of MpPAL genes was analyzed by semi-quantitative RT-PCR.

Fig. 6. Effects of a PAL inhibitor on the accumulation of luteolin (1), apigenin (2), and isoriccardin C (3) in wounded M. polymorpha.

M. polymorpha plants were grown on 1/2 Gamborg's B5 medium containing $1.4 \%$ agar for 30 days and then treated with $1 \mathrm{mM} \alpha$-aminooxy- $\beta$-phenylpropionic acid (AOPP), a PAL inhibitor. Plants that were subjected to wounding and AOPP treatment were harvested at the indicated times after the treatments. White bars, wounded plants; gray bars, wounded plants treated with AOPP. Data represent means \pm S.D. of three separate experiments. (Student's $t$-test, ${ }^{*} p<0.05,{ }^{* *} p<0.01$ ).

Fig. 7. Effects of wounding on ABA accumulation in M. polymorpha.

Plants were grown on 1/2 Gamborg's B5 agar medium containing $1.4 \%$ agar for 30 days and then subjected to wounding treatment. Plants were harvested at the indicated time. The ABA concentration was analyzed by UPLC-MS/MS. Data represent means \pm S.D. of three separate experiments.

Fig. 8. Effects of 12-oxo-phytodienoic acid on the concentration of isoriccardin $\mathrm{C}$ (3) in $M$. polymorpha.

M. polymorpha plants were grown on $1 / 2$ Gamborg's B5 medium containing $1.4 \%$ agar for 30 days. After treatment with $50 \mu \mathrm{M}$ 12-oxo-phytodiemoic acid (OPDA) for 1 hour, plants were 
harvested, and the concentration of isoriccardin C (3) was analyzed by HPLC. Data represent means \pm S.D. of three separate experiments. 
Figure 1. (Yoshikawa et al.)

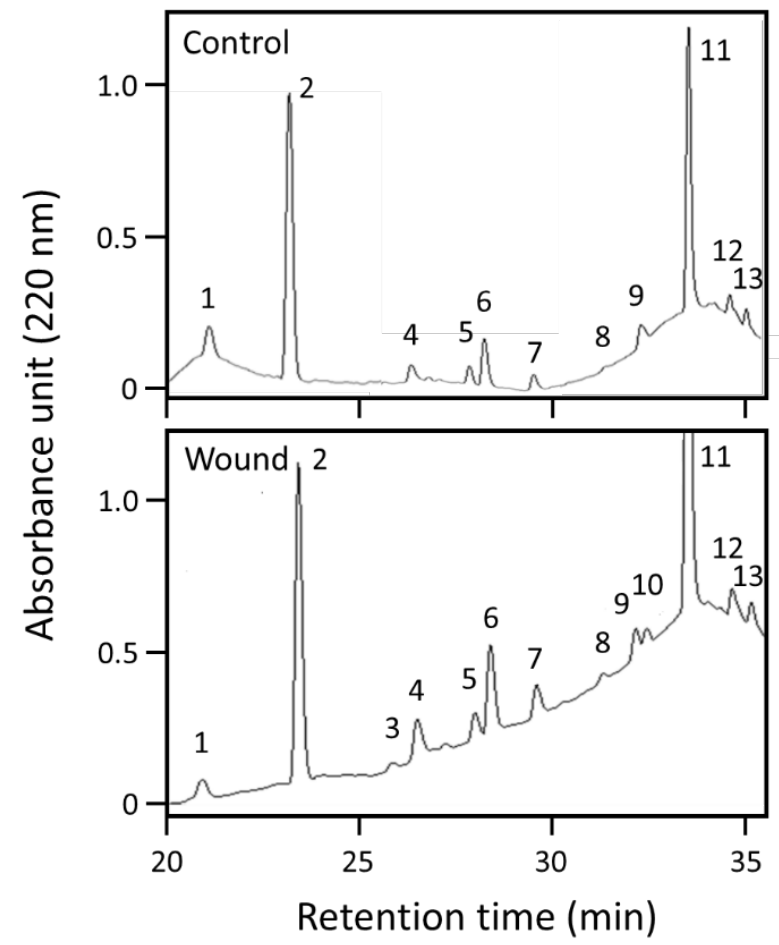


Figure 2. (Yoshikawa et al.)
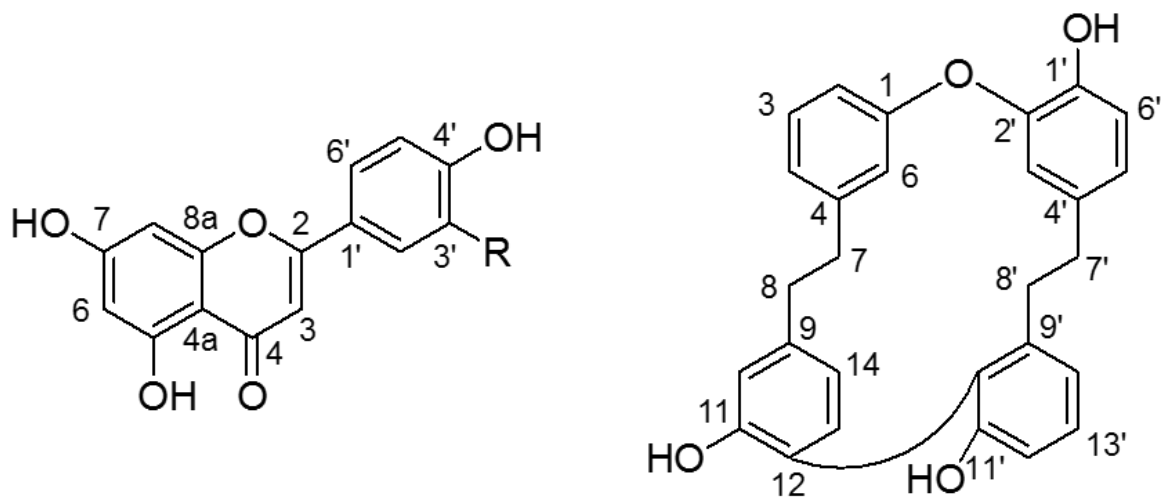

Luteolin (1) : $\mathrm{R}=\mathrm{OH}$

Apigenin (2): $\mathrm{R}=\mathrm{H}$

Isoriccardin C (3) 
Figure 3. (Yoshikawa et al.)
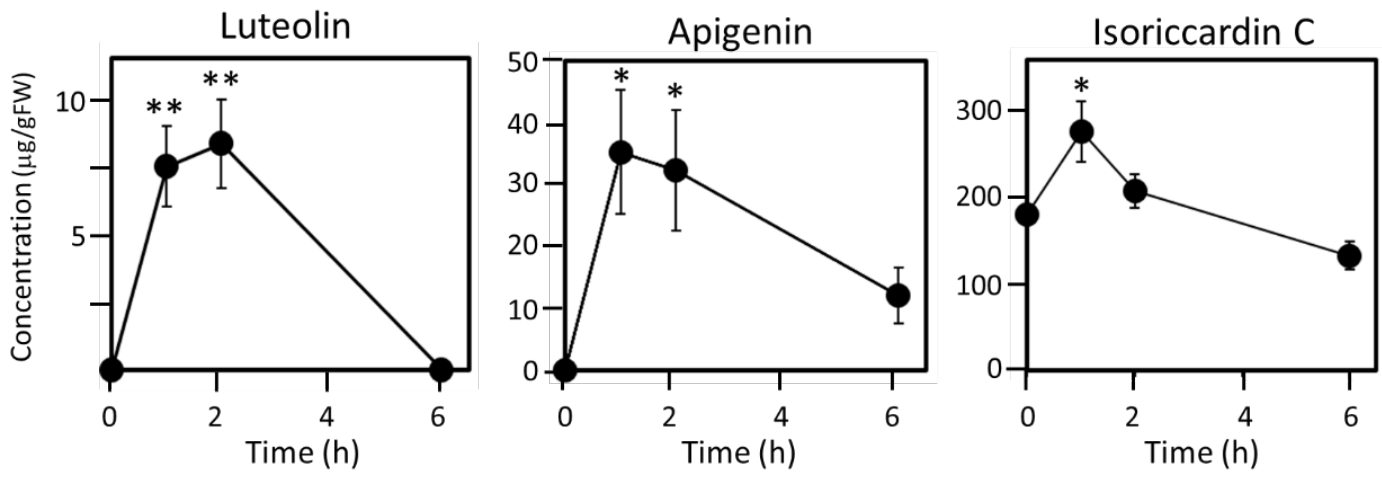
Figure 4. (Yoshikawa et al.)
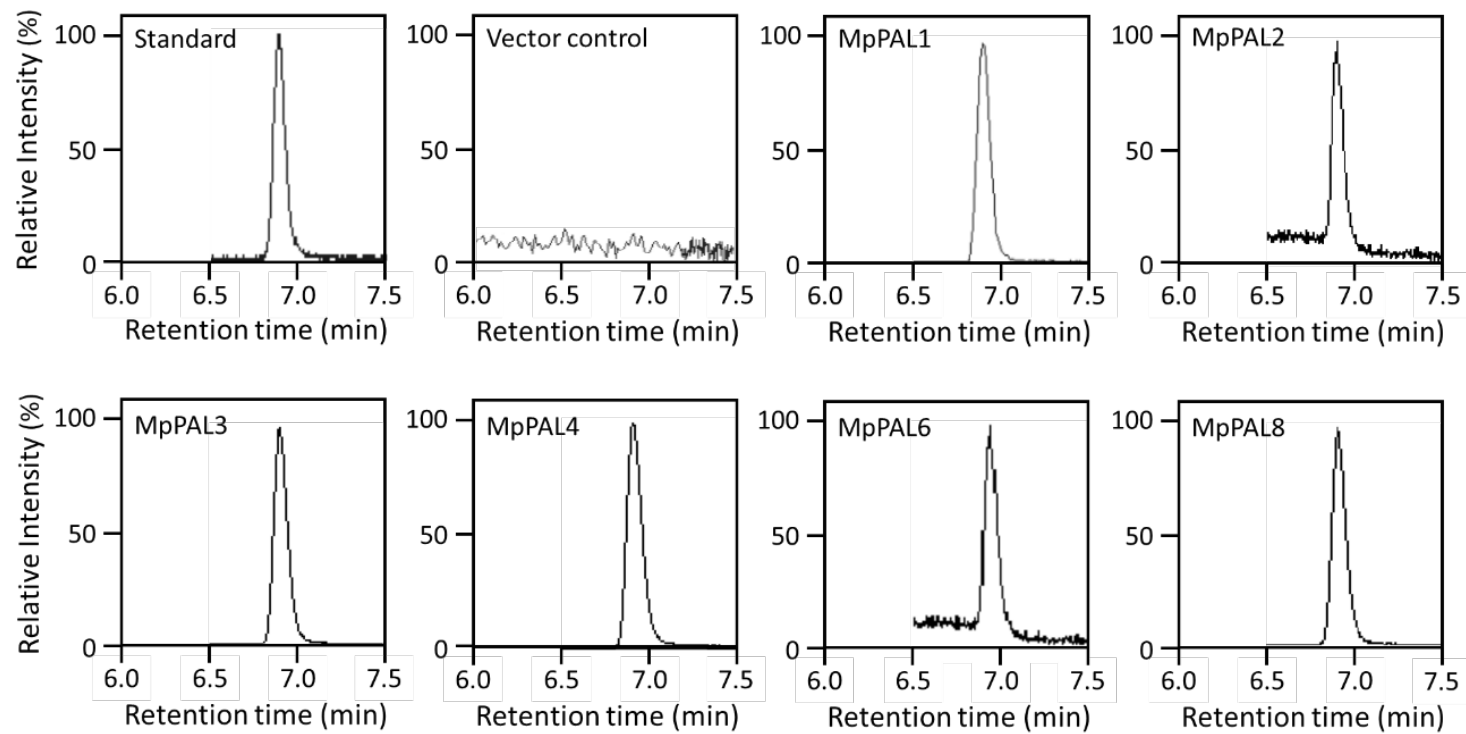
Figure 5. (Yoshikawa et al.)

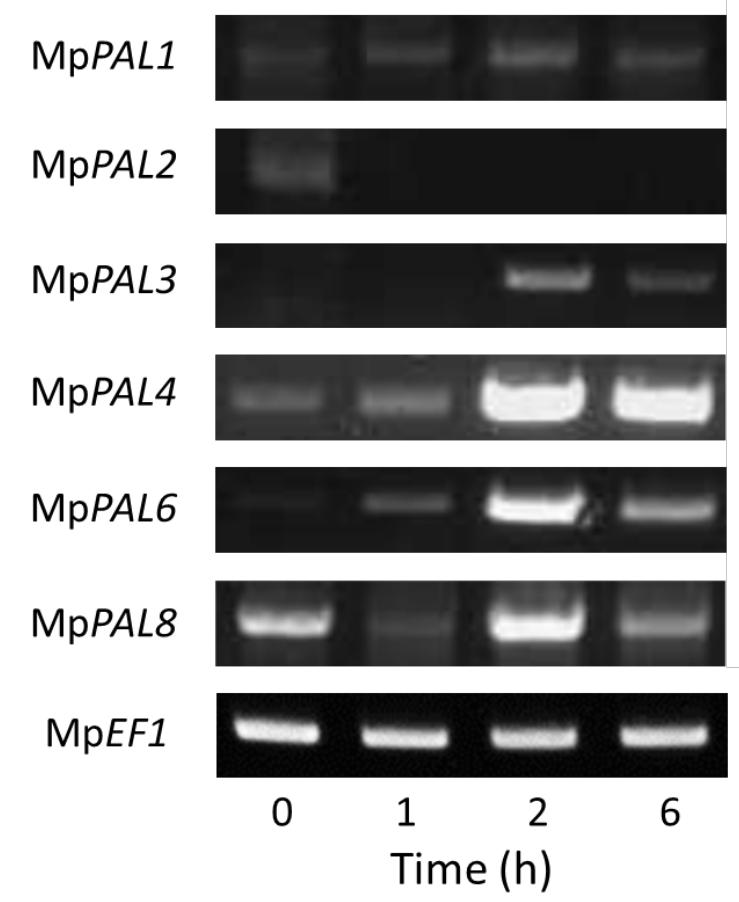


Figure 6. (Yoshikawa et al.)
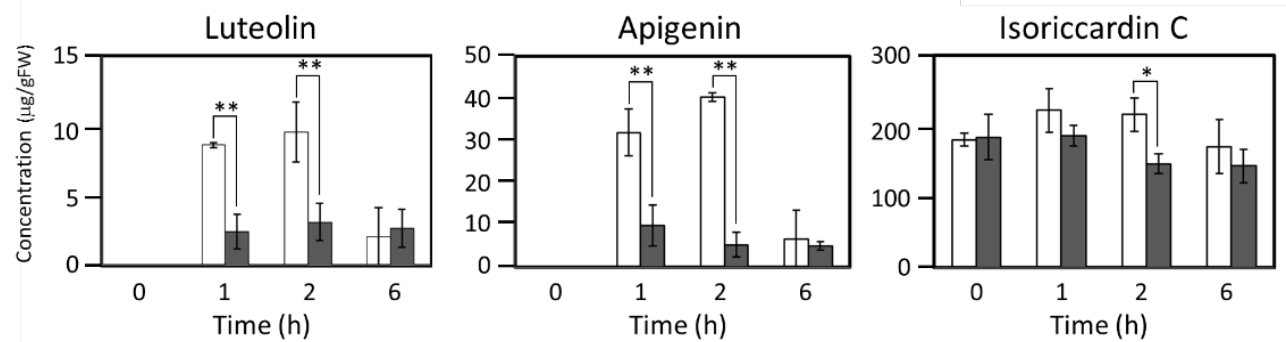
Figure 7. (Yoshikawa et al.)

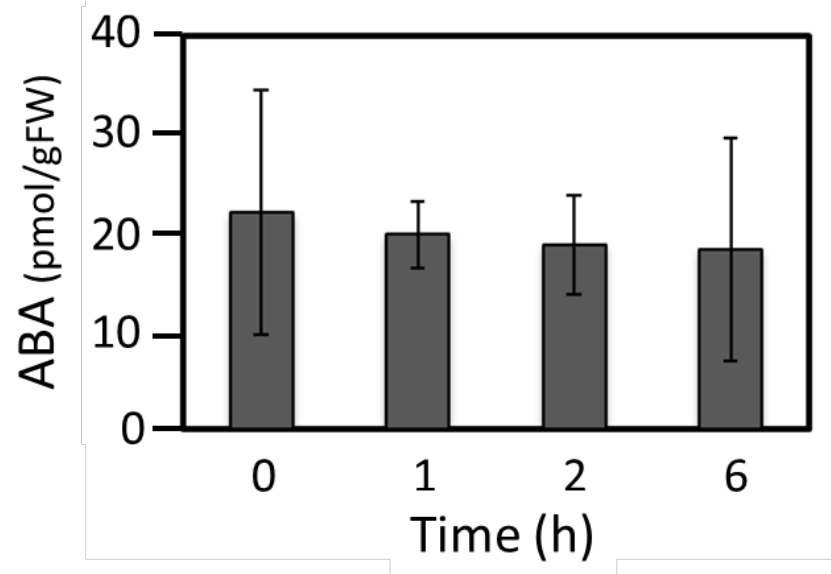


Figure 8. (Yoshikawa et al.)

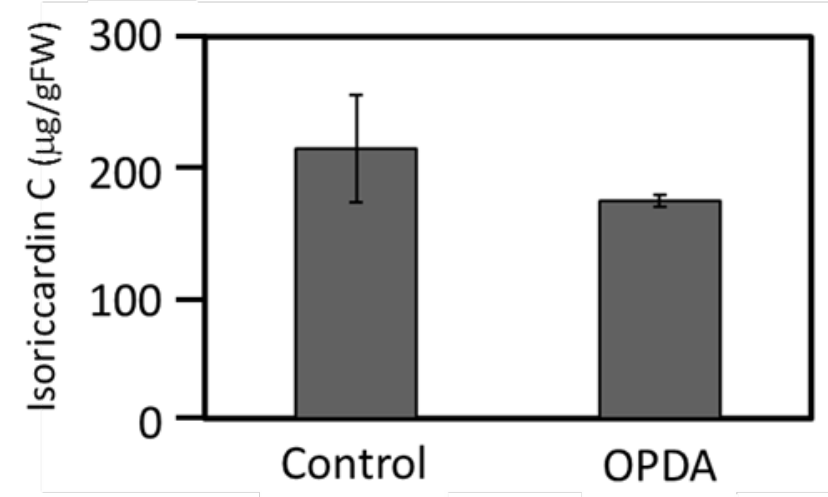




\section{Supplemental Data}

Wounding stress induces phenylalanine ammonia lyases, leading to the accumulation of phenylpropanoids in the model liverwort Marchantia polymorpha

Mayu Yoshikawa, Weifeng Luo, Genta Tanaka, Yuka Konishi, Hideyuki Matsuura, Kosaku Takahashi

Research Faculty of Agriculture, Hokkaido University, Sapporo, 060-8589, Japan

1. NMR spectral data of luteolin (1)

${ }^{1} \mathrm{H}$ NMR $\left(500 \mathrm{MHz}, \mathrm{CD}_{3} \mathrm{OD}\right): \delta 6.20(1 \mathrm{H}, \mathrm{d}, J=2.1 \mathrm{~Hz}, \mathrm{H}-8), 6.45(1 \mathrm{H}, \mathrm{d}, J=2.1 \mathrm{~Hz}, \mathrm{H}-6), 6.67$ $(1 \mathrm{H}, \mathrm{s}, \mathrm{H}-3), 6.90\left(1 \mathrm{H}, \mathrm{d}, J=8.1 \mathrm{~Hz}, \mathrm{H}-5\right.$ ') $7.40\left(1 \mathrm{H}, \mathrm{d}, J=2.3 \mathrm{~Hz}, \mathrm{H}-2^{\prime}\right), 7.42(1 \mathrm{H}, \mathrm{dd}, J=8.2,2.3$ Hz, H-6').

${ }^{13} \mathrm{C}$ NMR (125 MHz, CD 3 OD): $\delta 94.3$ (C-8), 99.2 (C-6), 103.2 (C-3), 104.1 (C-10), 113.8 (C-2'), 116.4 (C-5'), 119.3 (C-6'), 121.9 (C-1'), 146.1 (C-3’), 150.1 (C-4'), 157.7 (C-9), 161.9 (C-7), 164.3 (C-2), 164.5 (C-5), $182.0(\mathrm{C}-4)$.

2. NMR spectral data of apigenin (2)

${ }^{1} \mathrm{H}$ NMR $\left(500 \mathrm{MHz}, \mathrm{CD}_{3} \mathrm{OD}\right): \delta 6.19(1 \mathrm{H}, \mathrm{d}, J=2.1 \mathrm{~Hz}, \mathrm{H}-8), 6.47(1 \mathrm{H}, \mathrm{d}, J=2.1 \mathrm{~Hz}, \mathrm{H}-6), 6.76$ $(1 \mathrm{H}, \mathrm{s}, \mathrm{H}-3), 6.92(2 \mathrm{H}, \mathrm{d}, J=9.7 \mathrm{~Hz}, \mathrm{H}-2$ ' and H-6'), $7.92(2 \mathrm{H}, \mathrm{d}, J=9.7 \mathrm{~Hz}, \mathrm{H}-3$ ' and $\mathrm{H}-5$ ').

${ }^{13} \mathrm{C}$ NMR (125 MHz, CD $\left.3 \mathrm{OD}\right): \delta 94.3$ (C-8), 99.2 (C-6), 103.2 (C-3), 104.1 (C-10), 116.3 (C-2' and C-6'), 121.6 (C-2), 128.8 (C-3' and C-5'), 157.7 (C-5), 161.5 (C-4'), 161.8 (C-9), 164.1 (C-1'), 164.5 (C-7), 182.1 (C-4).

3. NMR spectral data of isoriccardin C (3) 
${ }^{1} \mathrm{H} \mathrm{NMR}\left(500 \mathrm{MHz}, \mathrm{CDCl}_{3}\right.$ ): $\delta 2.23$ (1H, m, H-7'a), 2.29 (1H, m, H-7’b), 2.49 (2H, m, H-8'), 3.09 (2H, m, H-8), 3.16 (2H, m, H-7), 5.57 (1H, d, J=1.0 Hz, H-3'), 6.62 (1H, dd, J = 8.1, $1.0 \mathrm{~Hz}, \mathrm{H}-14)$, $6.68\left(1 \mathrm{H}, \mathrm{dd}, J=8.1,1.0 \mathrm{~Hz}, \mathrm{H}-5\right.$ ') $, 6.75(1 \mathrm{H}, \mathrm{d}, J=1.0 \mathrm{~Hz}, \mathrm{H}-10), 6.82\left(1 \mathrm{H}, \mathrm{m}, \mathrm{H}-14^{\prime}\right), 6.83(1 \mathrm{H}$, m, H-6'), 6.86 (1H, m, H-2), 6.88 (1H, m, H-12'), 6.94 (1H, m, H-6), 6.95 (1H, m, H-13), 7.05 (1H, dd, $J=8.1,1.0 \mathrm{~Hz}, \mathrm{H}-3), 7.11$ (1H, dd, $J=8.1,1.0 \mathrm{~Hz}, \mathrm{H}-5), 7.26$ (1H, dd, $J=8.1,1.0 \mathrm{~Hz}, \mathrm{H}-13$ ').

${ }^{13} \mathrm{C}$ NMR (125 MHz, $\left.\mathrm{CDCl}_{3}\right): \delta 34.47\left(\mathrm{C}^{\prime} 8^{\prime}\right), 36.08$ (C-7), $36.50(\mathrm{C}-8), 37.95\left(\mathrm{C}^{\prime} 7^{\prime}\right), 113.24$ (C-12'), 114.58 (C-6), 114.64 (C-6'), 116.6 (C-10), 117.02 (C-2), 120.41 (C-3'), 121.60 (C-12), 121.64 (C-14'), 121.68 (C-14), 121.72 (C-5’), 122.59 (C-10'), 130.11 (C-5), 130.30 (C-3), 130.51 (C-13'), 130.81 (C-13), 133.60 (C-4'), 137.20 (C-4), 142.82 (C-9), 143.28 (C-9'), 143.59 (C-2'), 147.77 (C-1'), 153.26 (C-1), 153.43 (C-11), 153.99 (C-11'). 
Table S1. Primers used for cloning and semi-quantitative RT-PCR.

\begin{tabular}{ll}
\hline Primernames & Sequences (5' to 3 ') \\
\hline MpPAL1_F & TTGGGCAGGTCCAGCGCTGAGAAGA \\
MpPAL1_R & CTTCTTCTGCTGCTGCTGCTGCTGT \\
MpPAL2_F & ATGATGAACGAAATCTTCTCGTCCA \\
MpPAL2_R & TTATATTACGATTCTAGACGGCTCG \\
MpPAL3_F & ATGATGCAAGTGAGAGACTCGTCCA \\
MpPAL3_R & CTATACTACAATCCCGCAGTGGTCG \\
MpPAL4_F & ATGAACGAATTCTTCTCGTCCAACT \\
MpPAL4_R & TTACGCTATTGTTTTGGAAGGCTTG \\
MpPAL6_F & GCTTTCGATCGACCGAGCTTTCGAG \\
MpPAL6_R & CATCGCATGGCACAACGGATTCTGA \\
MpPAL8_F & GGCCTGAGGTTCGAGGCTGGGCGAC \\
MpPAL8_R & CCGCCGATCAGATTCATTCATTCCT \\
MpEF1_qPCR_F1 & AAGCCGTCGAAAAGAAGGAG \\
MpEF1_qPCR_R1 & TTCAGGATCGTCCGTTATCC \\
\hline
\end{tabular}


Table S2. Primers used for construction of vectors for recombinant protein production.

\begin{tabular}{ll}
\hline Primer names & Sequences (5' to 3 ') \\
\hline MpPAL1_OV_F & CATATGGTGGTCGAGACTGGAGCAAG \\
MpPAL1_OV_R & GCTCGAGTGAACGGGCCCGGGGCTCC \\
MpPAL2_OV_F & CATATGATGAACGAAATCTTCTCGTCCA \\
MpPAL2_OV_R & GCTCGAGTTATTACGATTCTAGACGGCT \\
MpPAL3_OV_F & CATATGATGCAAGTGAGAGACTCGTCCA \\
MpPAL3_OV_R & GCTCGAGTTACTACAATCCCGCAGTGGT \\
MpPAL4_OV_F & CATATGAACGAATTCTTCTCGTCCAACT \\
MpPAL4_OV_R & GCTCGAGCGCTATTGTTTTGGAAGGCT \\
MpPAL6_OV_F & CATATGATGAACGAATTCTTCTCATCGA \\
MpPAL6_OV_R & GCTCGAGTAACTACAATTTTGCAAGGCT \\
MpPAL8_OV_F & CATATGGCGGCCATGGTGATGGAGGTG \\
MpPAL8_OV_R & GCTCGAGTTACACGAGGCGCGTCGACG \\
\hline
\end{tabular}


Table S3. The annealing temperatures and the numbers of PCR cycles for semi-quantitative RT-PCR.

\begin{tabular}{lcc}
\hline Genes & Annealing temperatures & Numbers of PCR cycles \\
\hline $\mathrm{Mp} P A L 1$ & $63^{\circ} \mathrm{C}$ & 40 \\
$\mathrm{Mp} P A L 2$ & $54^{\circ} \mathrm{C}$ & 36 \\
$\mathrm{Mp} P A L 3$ & $60^{\circ} \mathrm{C}$ & 36 \\
$\mathrm{Mp} P A L 4$ & $63^{\circ} \mathrm{C}$ & 36 \\
$\mathrm{Mp} P A L 6$ & $63^{\circ} \mathrm{C}$ & 36 \\
$\mathrm{Mp} P A L 8$ & $60^{\circ} \mathrm{C}$ & 36 \\
$\mathrm{Mp} E F 1$ & $60^{\circ} \mathrm{C}$ & 40 \\
\hline
\end{tabular}




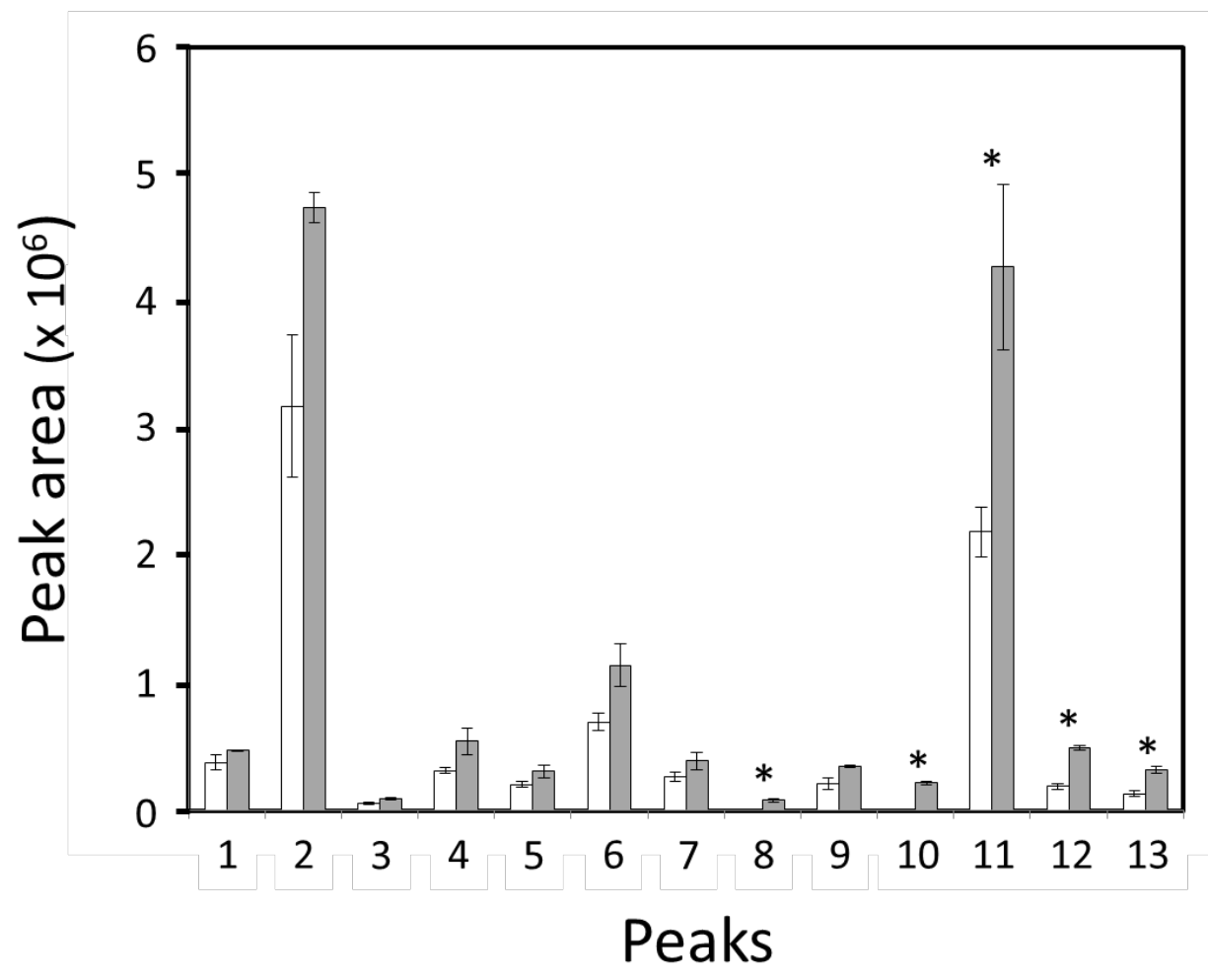

Fig. S1. Comparison of the peak areas from HPLC analyses of wounded M. polymorpha extract and untreated M. polymorpha extract.

White bars, control plants; gray bars, wounded plants. Data represent the means \pm S.D. of three separate experiments. The asterisks represent significant differences between wounded and control plants (Student's $t$-test, ${ }^{*} p<0.05$ ). 


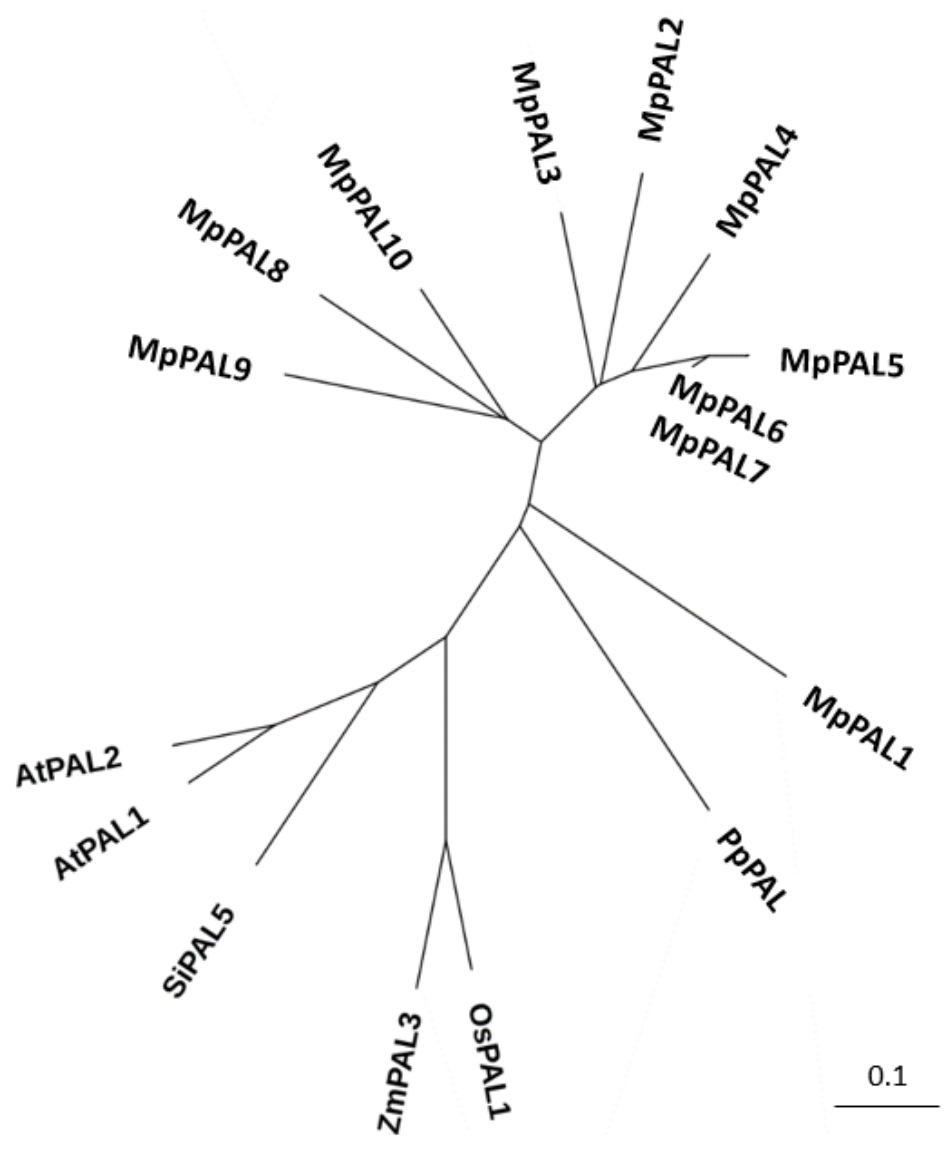

Fig. S2. Phylogenetic tree of the MpPALs and previously reported PALs.

The phylogenetic tree was constructed using Clustal Omega (version 1.2.4) with the following: MpPAL1 (Mapoly0014s0211.1), MpPAL2 (Mapoly0044s0114.1), MpPAL3 (Mapoly0009s0173.1), MpPAL4 (Mapoly0070s0061.1), MpPAL5 (Mapoly0070s0065.1), MpPAL6 (Mapoly0070s0068.1), MpPAL7 (Mapoly0070s0071.1), MpPAL8 (Mapoly0132s0049.1), MpPAL9 (Mapoly0005s0086.1), MpPAL10 (Mapoly0005s0089.1), AtPAL1 (Arabidopsis thaliana, AY303128), AtPAL2 (Arabidopsis thaliana, AY303129), OsPAL1 (Oryza sativa, AY224546), SiPAL5 (Solanum lycopersicum, P26600), ZmPAL3 (Zea mays, NM_001111864) and PpPAL (Physcomitrella patens, XM_001785612) 


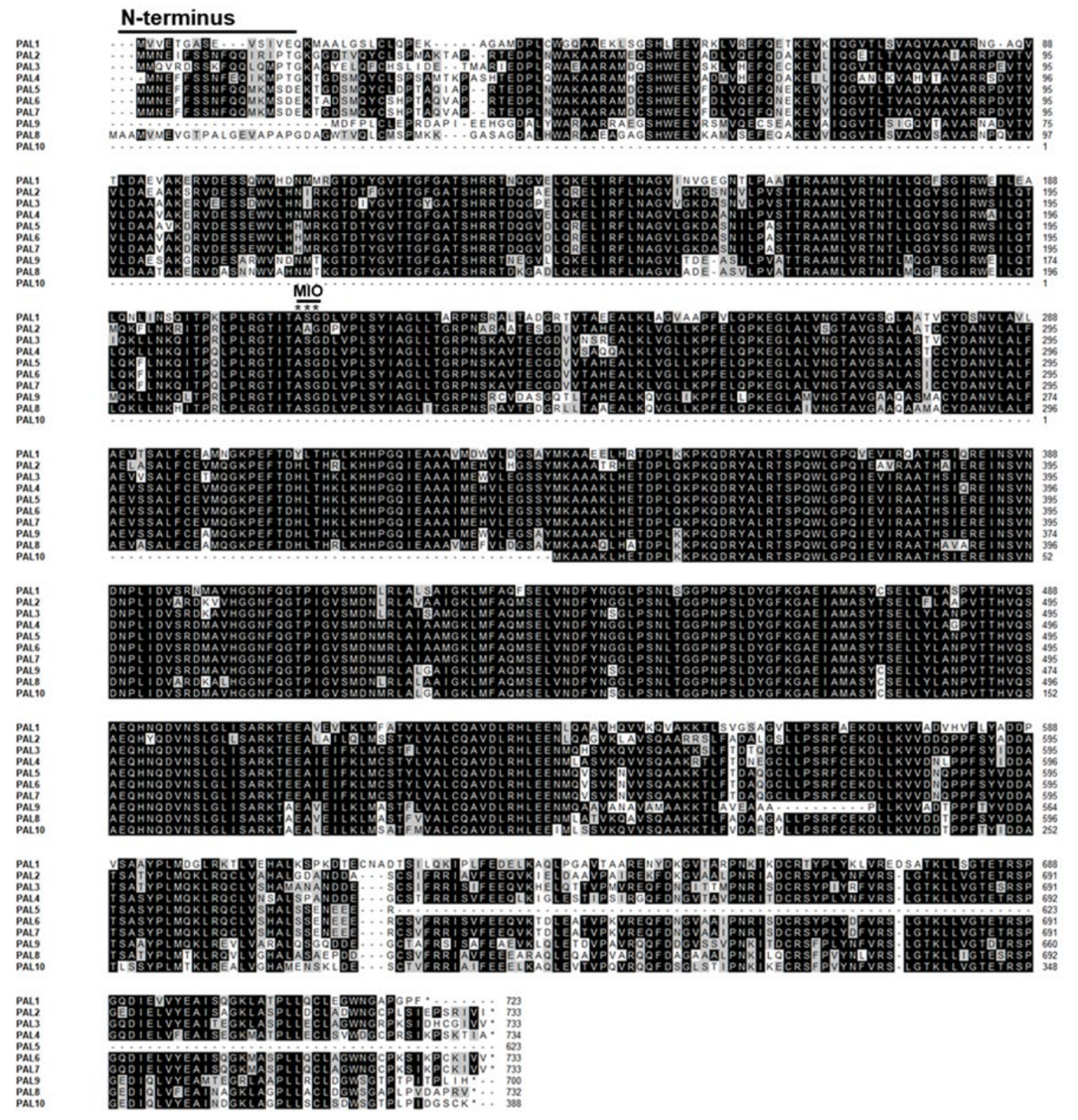

Fig. S3. Amino acid sequence alignment of the MpPALs.

The amino acid sequences were aligned using Clustal Omega (version 1.2.4). Identical and similar amino acids are highlighted in black and gray, respectively. The aligned sequences are MpPAL1 (Mapoly0014s0211.1), MpPAL2 (Mapoly0044s0114.1), MpPAL3 (Mapoly0009s0173.1), MpPAL4 (Mapoly0070s0061.1), MpPAL5 (Mapoly0070s0065.1), MpPAL6 (Mapoly0070s0068.1), MpPAL7 (Mapoly0070s0071.1), MpPAL8 (Mapoly0132s0049.1), MpPAL9 (Mapoly0005s0086.1), 
and MpPAL10 (Mapoly0005s0089). MIO indicates the conserved active site amino acid sequence Ala-Ser-Gly in PALs. Cyclization of Ala-Ser-Gly produces the cofactor of the PAL reaction, 3,5-dihydro-5-methylidene-4H-imidazole-4-one (MIO). 